\title{
Los censos visuales en transectos de banda subestiman la abundancia del pez león Pterois spp. en arrecifes coralinos del Caribe
}

\author{
Visual censuses on belt transects underestimate the abundance of lionfish Pterois spp. \\ on Caribbean coral reefs

\section{Miguel Mateo Sabido-Itzá ${ }^{*}$, José Manuel Castro-Pérez', Gabriela Nava-Martínez², Alejandro Medina-Quej ${ }^{1}$ y Martha Beatriz Hernández-Millán ${ }^{1}$}

\author{
'Tecnológico Nacional de México, I.T. Chetumal, Av. Insurgentes No. 330, C.P. 77013, Chetumal, Quintana Roo, México, Av. Insurgentes \\ No. 330, C.P. 77013, Chetumal, Quintana Roo, México \\ ${ }^{2}$ OCEANUS AC, Av. Machuxac, lote 07, Mza. 235, C.P. 77086, Chetumal, Quintana Roo, México \\ *Autor corresponsal: mateosabido@gmail.com
}

\begin{abstract}
The visual census on belt transect (CVB) is one of the most used monitoring protocol on reefs, however this study confirms that its application fail in the detection and estimation of abundance of invasive lionfish. CVB vs Lionfish Search Census (CBP) was compared on Mexican Caribbean reefs. Differences in abundance of lionfish were found between CVB and CBP. Bias among both methods was significantly correlated to the cryptic behavior of inactivity and depth of the site. Finally, the use of different lionfish sampling methods and the application of CBP for monitoring reefs in the region are discussed.
\end{abstract}

Key words: Visual census, lionfish, Mexican Caribbean, Mesoamerican Reef System

\section{INTRODUCCIÓN}

El censo visual de transecto de banda es uno de los protocolos más utilizados para la evaluación de la riqueza, densidad y biomasa de peces arrecifales (Brock 1982), este método tiene la ventaja de ser económico, no destructivo, generalmente rápido y fácilmente adaptable (Sanderson \& Solonsky 1986, Samoilys \& Carlos 2000, Bacheler et al. 2017). Sin embargo, generalmente se enfoca a las comunidades superficiales del arrecife, subestimando las abundancias de especies con comportamiento críptico y de hábitos nocturnos (Willis 2001, Azzurro et al. 2007). Estos errores pueden repercutir en la subestimación de las densidades de especies focales de las cuales es necesario contar con una mayor exactitud de sus poblaciones que permita proveer de información confiable para acciones de manejo (Feary et al. 2011, Reyes-Bonilla et al. 2016, Kayal et al. 2017). Un ejemplo de ello es el pez león (Pterois volitans / P. miles), cuya invasión ha alcanzado la costa este de EUA, mar Caribe y golfo de México (Schofield 2010). Debido a su rápido crecimiento corporal, alta fecundidad y carencia de depredadores (Côté et al. 2013), ha propiciado un aumento de sus poblaciones en diferentes hábitats (Claydon et al. 2012), incluso superando las de su área de distribución natural (Darling et al. 2011). Esto se traduce en la afectación de la red trófica del arrecife (AriasGonzález et al. 2011), como consecuencia de su apetito voraz y consumo generalista de peces e invertebrados de importancia ecológica y comercial (Arredondo-Chávez et al. 2016). Ante estos potenciales impactos, se ha priorizado la estimación de las abundancias del pez león a través de la implementación de diferentes metodologías tales como la aplicación de Rotenona (Kulbicki et al. 2012), video-transectos (Andradi-Brown et al. 2017a, Bacheler et al. 2017), calcular la Captura Por Unidad de Esfuerzo (CPUE) (Frazer et al. 2012), marcaje y recaptura (Akins et al. 2014), método de distancia (Tilley et al. 2016) y censos visuales; siendo estos últimos los más utilizados (Darling et al. 2011, Green et al. 2013, Cure et al. 2014, Hackerott et al. 2017, Peiffer et al. 2017).

En la región del Sistema Arrecifal Mesoamericano (SAM), el pez león, considerado una verdadera amenaza para los arrecifes coralinos (Gómez-Lozano et al. 2013) desde su primer registro en 2008, su población ha sido evaluada con censos visuales de banda para peces arrecifales (HRI 2012). No obstante, se ha documentado que la aplicación de este método puede subestimar la abundancia real dado que no considera la naturaleza críptica de la especie, además de las condiciones físicas del arrecife (Kulbicki et al. 2012, Green et al. 2013). Por tal motivo, actualmente se utilizan censos dirigidos hacia la búsqueda específica del pez león con la finalidad de obtener resultados más precisos (Anderson et al. 2017, Hackerott et al. 2017, Sabido-Itzá \& García-Rivas 2019). 
El objetivo de este trabajo fue comparar el grado de detección y la estimación de la abundancia del pez león entre dos tipos de censos visuales: censo visual en transecto de banda (CVB) y censo de búsqueda de pez león (CBP). Además, correlacionar la diferencia en la abundancia de pez león entre los CVB y CBP con variables ambientales tal como la complejidad topográfica y profundidad, y con variables biológicas de la especie como el comportamiento críptico de inactividad y talla. Recientemente se reportó la presencia de $P$. miles en el área de estudio pero su distinción respecto a $P$. volitans durante las observaciones es muy difícil dada su sorprendente similitud morfológica, por lo que pez león y Pterois spp. se utilizan en referencia a estas especies (Guzmán-Méndez et al. 2017). Finalmente, el presente trabajo pretende discutir las implicancias de las metodologías de muestreo en la región del SAM.

\section{MATERIALES Y MÉTODOS}

Los monitoreos se realizaron entre octubre y noviembre de 2014 en 5 sitios por cada una de las 3 localidades arrecifales del Caribe mexicano: Xcalak $\left(18^{\circ} 30^{\prime}-18^{\circ} 11^{\prime} \mathrm{N}, 87^{\circ} 43^{\prime}\right.$ $\left.87^{\circ} 50^{\prime} \mathrm{W}\right)$, Mahahual (18 $48^{\circ} 4^{\prime}-18^{\circ} 37^{\prime} \mathrm{N}, 87^{\circ} 40^{\prime}-87^{\circ} 43^{\prime} \mathrm{W}$ ) y Banco Chinchorro (18 $47^{\circ}$ '-18 $\left.23^{\circ} \mathrm{N}, 87^{\circ} 14^{\prime}-87^{\circ} 27^{\prime} \mathrm{W}\right)$. Estas localidades se encuentran en la porción sur del estado de Quintana Roo dentro de la sección norte del Sistema Arrecifal Mesoamericano (SAM), considerados como sitios prioritarios para el Programa de Monitoreo Sinóptico del SAM (Almada-Villela et al. 2003) debido a su alta biodiversidad (Arias-González 1998, Kramer \& Kramer 2002).

Se aplicaron dos métodos de muestreo de transecto de banda (CVB y CBP) para comparar el grado de detección y la estimación de la abundancia del pez león a través de transectos de $30 \times 4 \mathrm{~m}\left(120 \mathrm{~m}^{2}\right)$ a $18,5 \mathrm{~m}$ de profundidad en promedio. Un primer buzo registró el número y talla en $\mathrm{cm}$ de los peces observados mientras realizaba un censo visual. Seguidamente sobre el mismo transecto, un segundo buzo, realizó una búsqueda minuciosa en oquedades, cornisas y grietas registrando la profundidad de cada transecto $(\mathrm{m})$, número de organismos, talla en $\mathrm{cm}$ y el comportamiento activo o inactivo (críptico) de cada pez león siguiendo las recomendaciones de Green et al. (2011), Green (2012) y García-Rivas et al. (2017). Debido a que el pez león usualmente es inactivo durante el día y no escapa ante la proximidad de los buzos (Green et al. 2013), es poco probable que se haya desplazado entre el intervalo del censo visual y la búsqueda dirigida. Dependiendo de la profundidad, se realizaron entre 3 y 4 censos por método utilizado en cada sitio; siendo un total de 114 transectos (57 CVB y 57 CBP).
La complejidad topográfica fue registrada desplazando una cadena de $10 \mathrm{~m}$ siguiendo el contorno del arrecife (Risk 1972). La cadena se desplegó sobre la cinta métrica registrando la distancia recorrida de la misma. La complejidad se determinó de la siguiente manera:

$$
\mathrm{CT}=1-\mathrm{D} / \mathrm{L}
$$

Dónde: CT es la complejidad topográfica, D es la distancia que recorrió la cadena siguiendo el contorno del arrecife y L es la longitud de la cadena completamente extendida $(10 \mathrm{~m})$.

Los censos para cada método fueron realizados por las mismas personas, durante el día entre 09:00 y 16:00 h, aprovechando la visibilidad de la luz solar.

Se calculó el porcentaje de ocurrencia del pez león por el tipo de método empleado:

$$
\% \mathrm{O}=(\mathrm{NCP} / \mathrm{NTC}) * 100
$$

Donde, \%O: porcentaje de ocurrencia, NCP: Número de censos con pez león y NTC: Número total de censos de cada método.

La detección del pez león por medio de los CVB, se consideró como el número de transectos de CVB que presentaron por lo menos un pez león cuando uno o más organismos fueron observados por el CBP en la misma área.

La abundancia de pez león fue cuantificada como el número de peces observados en cada censo. Se utilizó la prueba de Wilcoxon de muestras pareadas para determinar si hubo diferencia significativa entre las abundancias de los CVB y CBP. Asimismo se utilizó un Análisis de la Varianza Simple (ANOVA) seguido de una prueba post-hoc de Rangos Múltiples (LSD-Fisher) para detectar diferencias entre localidades de monitoreo (Zar 1999). Los datos fueron transformados a $\log (\mathrm{x}+1)$, con el propósito de cumplir con el supuesto de normalidad (Prueba ShapiroWilk) y homocedasticidad (Prueba Levene) (Zar 1999).

Para evaluar la relación de variables físicas del arrecife y biológicas de la especie con respecto a la subestimación del pez león, se utilizó el sesgo de los CVB, calculado a partir de la diferencia en la abundancia de pez león entre el CVB y $\mathrm{CBP}$; estos valores fueron utilizados para correlacionarlos con la complejidad topográfica, profundidad, talla y número de peces que tuvieron un comportamiento críptico de inactividad a través de una matriz de Pearson. 


\section{RESULTADOS Y DISCUSIÓN}

Los Censos de Búsqueda de Pez león (CBP) tuvieron un $70 \%$ de ocurrencia de Pterois spp. (40 censos), mientras que los Censos Visuales en transecto de Banda (CVB) presentaron un $37 \%$ de ocurrencia (21 censos). Los CBP registraron 85 individuos, en tanto que los CVB solo 26 individuos; este último método de muestreo logró detectar únicamente el $31 \%$ de los peces registrados por los CBP. Además, el 63\% de los CVB fallaron en la detección de la presencia del algún pez león tomando como referencia los $\mathrm{CBP}$ en la misma área. Estos resultados son muy similares a los reportados por Green et al. (2013) en los arrecifes de Bahamas, donde mencionan que solo el $28 \%$ de los peces fueron observados y el $64 \%$ de los CVB fallaron en la detección de al menos un pez león.

La abundancia en los CBP tuvieron un rango de 0 a 8 individuos (media $\pm \mathrm{DE} ; 1,49 \pm 1,67$ ind. $120 \mathrm{~m}^{-2}$ ), mientras que en los CVB fueron de 0 a 3 individuos (media $\pm \mathrm{DE}$; $0,45 \pm 0,68$ ind. $120 \mathrm{~m}^{-2}$ ). La abundancia de Pterois spp. en los CBP fue significativamente mayor en un orden de magnitud de 2,3 veces, respecto a la abundancia de los CVB $(Z=4,97 ; P<0,0001)$. Los CVB, han sido utilizados frecuentemente en la estimación de las densidades de pez león; sin embargo, el porcentaje de ocurrencia en los mismos han sido muy bajos (Kulbicki et al. 2012, Ruttenberg et al. 2012), probablemente subestimando la abundancia real y por ende minimizando los efectos que pudieran ocurrir por la presencia de altas densidades de las poblaciones de este pez en los sistemas arrecifales.
Se ha demostrado que algunos atributos poblaciones del pez león, como la estructura de tallas, relación longitudpeso y factor de condición, difieren ligeramente entre las localidades estudiadas, asociado principalmente a la disponibilidad de alimento (Sabido-Itzá et al. 2016a). Sin embargo, la abundancia no varió significativamente entre las localidades de monitoreo $\left(\mathrm{F}_{2,54}=1,12, P=0,33\right)$ (Fig. 1), debido probablemente a que su invasión y establecimiento fue homogéneo en los arrecifes de la porción sur de Quintana Roo (Sabido-Itzá et al. 2016b), lo cual le permitió tener abundancias constantes en la zona de estudio.

La diferencia en abundancia de pez león entre ambos tipos de censos, aquí referido como el sesgo de los CVB, no tuvo una correlación significativa con la complejidad topográfica del arrecife ni con la talla (Tabla 1). Estos datos contrastan con los reportados por Green et al. (2013), debido a que contemplaron las zonas arrecifales más someras y de menor complejidad estructural de la laguna arrecifal, donde se encuentra mayor proporción de peces juveniles (Claydon et al. 2012), lo cual limita su detección. En contraste, en este estudio los censos estuvieron restringidos al arrecife frontal, con profundidades $>10$ $\mathrm{m}$ y con una complejidad topográfica relativamente homogénea (Tabla 1). Sin embargo, el sesgo de los CVB se correlacionó significativamente con el número de peces que presentaron un comportamiento inactivo y que estuvieron resguardados dentro del arrecife, así como en menor medida con la profundidad de los sitios. Debido a que los monitoreos de este trabajo fueron realizados durante

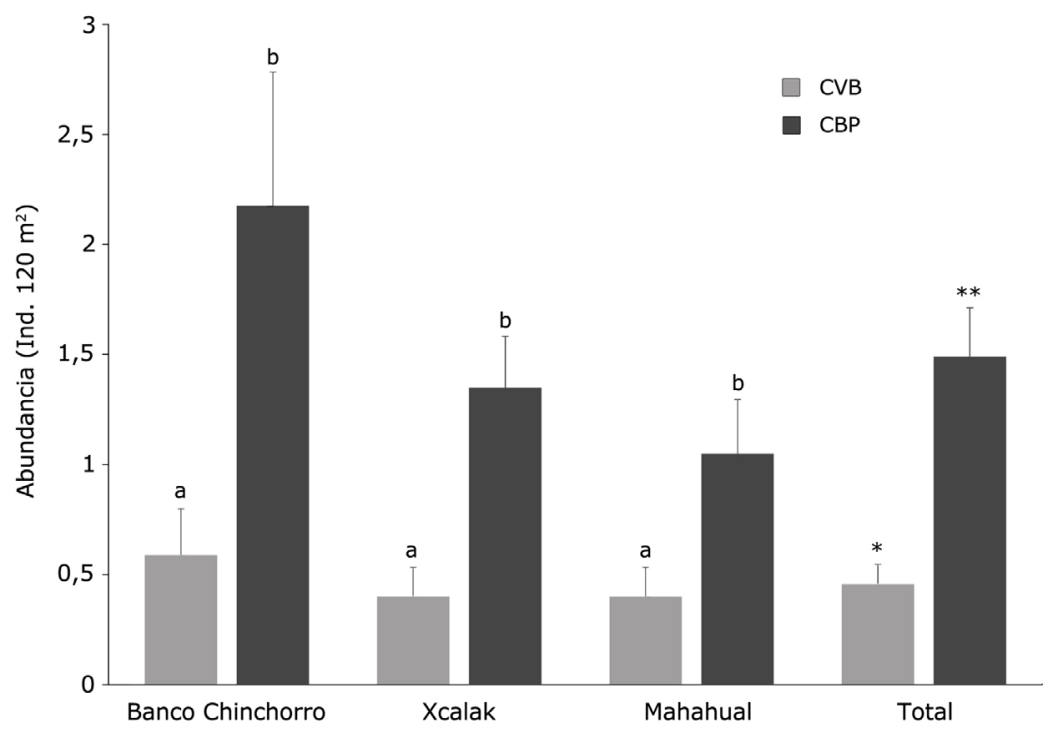

Figura 1. Comparación de la abundancia del pez león a través de dos tipos de métodos de muestreo: Censo Visual en transecto de Banda (CVB) (promedio \pm Error estándar) y Censo de Búsqueda de Pez león (CBP) en tres localidades arrecifales del Caribe mexicano. Las letras indican los grupos homogéneos y los asteriscos diferencia significativa entre los tipos de monitoreo $P<0,05 /$ Comparison of the abundance (mean \pm standard error) of lionfish through two types of sampling methods: Visual Censuses on Belt transect (CVB) and Lionfish Search Census (CBP) at three reef locations in the Mexican Caribbean. The letters indicate the homogeneous groups, and the asterisks significant difference between the sampling types $P<0.05$ 
Tabla 1. Coeficientes de correlación de Pearson entre el sesgo de los tipos de censos con las variables físicas (complejidad topográfica y profundidad) y biológicas (talla y comportamiento de inactividad) / Pearson's correlation coefficient between the bias of census types and physical variables (topographical complexity and depth) as well as biological variables (size and inactivity behavior)

\begin{tabular}{lcc}
\hline & \multicolumn{2}{c}{$\begin{array}{c}\text { Sesgo de la abundancia de } \\
\text { pez león entre CVB y CBP }\end{array}$} \\
\cline { 2 - 3 } Variables (media \pm D.E.) & $r$ & $P$ \\
\hline Físicas del hábitat & & \\
Complejidad topográfica $(0,34 \pm 0,14)$ & 0,041 & 0,771 \\
Profundidad (18,49 $\pm 4,24 \mathrm{~m})$ & 0,313 & $0,018^{*}$ \\
Biológicas de la especie & & \\
Longitud total $(25,11 \pm 7,46 \mathrm{~cm})$ & 0,066 & 0,69 \\
Comportamiento inactivo $(51 \%$ ind.) & 0,816 & $<0,001^{*}$ \\
\hline
\end{tabular}

$\mathrm{CVB}=$ Censo Visual en transecto de Banda, $\mathrm{CBP}=$ Censo de Búsqueda de Pez león, D.E: Desviación estándar, *indica relación significativa $P<0,05$

el día, los resultados son consistentes al comportamiento típico del pez león en el Atlántico y área nativa, ya que la mayor actividad de caza y desplazamiento se asocia a los periodos crepusculares (amanecer-anochecer) y menor a mediodía (Green et al. 2011, Cure et al. 2012). En el Caribe mexicano, García-Rivas et al. (2017) encontraron que los peces estudiados durante el día estuvieron mayormente inactivos y refugiados dentro de cuevas. Respecto a la profundidad, Tilley et al. (2016), probaron que la probabilidad de detección del pez león fue mayor en zonas someras $(<15 \mathrm{~m})$ y esta disminuye significativamente en las zonas profundas del arrecife $(>15 \mathrm{~m})$, influenciado quizá por la rugosidad del arrecife o visibilidad del mar. En este sentido, se recomienda seguir aplicando los CBP o bien implementar monitoreos en periodos crepusculares con la finalidad de disminuir el sesgo que produce el comportamiento críptico del pez león y obtener registros de abundancias más precisos que puedan ser utilizados en las acciones de manejo y control.

\section{IMPLICACIONES DE MONITOREO EN EL SAM}

Desde el primer avistamiento del pez león en la región del SAM y su establecimiento en el Caribe mexicano (SabidoItzá et al. 2012), se ha reportado que esta especie consume un total de 54 familias de peces y crustáceos (Rocha et al. 2015, Arredondo-Chávez et al. 2016, Bogdanoff et al. 2018). Estos resultados colocan en riesgo a especies de importancia comercial, ecológica y endémica, consideradas como prioritarias en la conservación.
Ante tal gravedad, en el 2014, se creó el 'Comité Regional del Arrecife Mesoamericano para el Control del Pez León' (Rodríguez et al. 2014) y uno de sus objetivos es la homologación de técnicas adecuadas para el monitoreo y control de las poblaciones de la especie. Con base en este estudio, el pez león debe ser incluido como un indicador clave en los monitoreos del arrecife, y asi obtener datos con mayor exactitud para focalizar los esfuerzos de control en sitios prioritarios, considerando el aumento en sus poblaciones y los efectos negativos en el arrecife (Albins 2015).

En la región del SAM se han implementado diferentes métodos para la estimación de las abundancias del pez león, destacando los CBP (Tabla 2). Por ejemplo, la metodología del Programa de Evaluación Rápida de Arrecifes del Atlántico y Golfo (AGRRA) contempla al pez león dentro de los censos convencionales de peces arrecifales en $60 \mathrm{~m}^{2}$ y dentro de los conteos de invertebrados clave en $20 \mathrm{~m}^{2}$ (Lang et al. 2012), no obstante, es muy probable obtener una subestimación de las densidades reales, ya que se registra únicamente a los peces que se encuentran superficialmente en el arrecife, aunado a que el área de muestreo se considera pequeña debido a su comportamiento gregario (Green 2012, García-Rivas et al. 2017). Asimismo, referente a la amplitud del transecto, Green (2012) propuso censos de búsqueda de 25 x $10 \mathrm{~m}$, mientras que Hackerott et al. (2017) utilizaron censos de $50 \times 10 \mathrm{~m}$. Para que el área de los transectos mencionados sean revisados en su totalidad, es necesario la supervisión de dos buzos los cuales al efectuar el recorrido aumentan los costos del monitoreo y tiempo de realización (Darling et al. 2011, Green 2012, Cure et al. 2014). Kulbicki et al. (2012) y Tilley et al. (2016) observaron un menor rango de error en la detección del pez león con una amplitud aproximada de $3 \mathrm{~m}$ de ancho de banda del transecto. Por lo tanto, se considera que la amplitud de los CBP empleados en este trabajo podrían ser fácilmente implementados en los monitoreos arrecifales realizados actualmente en el SAM, debido a que ya se cuenta con una línea base dentro del protocolo de monitoreo sinóptico por más de 10 años y que contempla un área de muestreo similar (GarcíaSalgado et al. 2006, HRI 2012). Además, el personal de las Áreas Marinas Protegidas de Cozumel, Xcalak y Banco Chinchorro, han aplicado la amplitud del transecto de $4 \mathrm{~m}$ obteniendo densidades de pez león que pueden ser comparadas espacial y temporalmente (Sabido-Itzá \& García-Rivas 2019). 
Tabla 2. Tipos de métodos de muestreo implementados para la estimación de la abundancia del pez león en la región del Sistema Arrecifal Mesoamericano (SAM) / Types of sampling methods implemented for estimating the abundance of lionfish in the Mesoamerican Reef System (SAM)

\begin{tabular}{|c|c|c|c|c|c|}
\hline $\begin{array}{l}\text { Tipo de } \\
\text { muestreo }\end{array}$ & País & $\begin{array}{l}\text { Rango de } \\
\text { profundidad } \\
(\mathrm{m})\end{array}$ & $\begin{array}{l}\text { Tamaño de } \\
\text { transecto } \\
(\mathrm{m})\end{array}$ & $\begin{array}{l}\text { Año de } \\
\text { monitoreo }\end{array}$ & Fuente \\
\hline Censo visual & México y Belice & $10-15$ & $50 \times 10$ & 2012 & Hackerott et al. 2013 \\
\hline Censo visual & Belice & $1-30$ & $30 \times 5$ & $2014-2015$ & Anderson et al. 2017 \\
\hline Censo visual & Honduras & 13 & $30 \times 2$ & 2012 & Andradi-Brown et al $2017 \mathrm{a}$ \\
\hline Censo visual & Belice & $12-15$ & $50 \times 10$ & $2009-2013$ & Hackerott et al. 2017 \\
\hline Censo visual & Honduras & 15 & $50 \times 5$ & 2015 & Peiffer et al. 2017 \\
\hline Censo visual & México & -- & $30 \times 4$ & 2013 & $\begin{array}{l}\text { Sabido-Itzá \& García-Rivas } \\
2019\end{array}$ \\
\hline $\begin{array}{l}\text { Censo Visual } \\
\text { Estacionario }\end{array}$ & México & $12-15$ & $5 \mathrm{~m}$ de radio & $2014-2015$ & Cobián-Rojas et al. 2018 \\
\hline Video transecto & Honduras & 45 & $50 \times 2,5$ & 2014 & Andradi-Brown et al. $2017 \mathrm{~b}$ \\
\hline Video transecto & Honduras & $5-85$ & $50 \times 5$ & 2015 & Andradi-Brown et al. $2017 \mathrm{~b}$ \\
\hline APUE & Belice & -- & -- & 2011-2015 & Anderson et al. 2017 \\
\hline CPUE & México, Belice y Honduras & -- & -- & $2010-2015$ & Malpica-Cruz et al. 2016 \\
\hline CPUE & Honduras & -- & -- & $2014-2015$ & Peiffer et al. 2017 \\
\hline CPUE & Belice & $15-35$ & -- & -- & Usseglio et al. 2017 \\
\hline
\end{tabular}

APUE $=$ Avistamientos Por Unidad de Esfuerzo, CPUE $=$ Captura Por Unidad de Esfuerzo

Por tanto, otros estudios y el presente han demostrado que el tipo de comportamiento del pez león sumado a las características del hábitat pueden sesgar las abundancias de peces en los CVB y de acuerdo a ello es importante contar con una metodología estandarizada que permita evaluar la expansión y cambios en la abundancia del pez león y de las comunidades nativas (Pieffer et al. 2017). El empleo de cualquier método dependerá en gran medida del interés de los manejadores y de la exactitud en que se pretenda estimar las abundancias del pez león, sin embargo, es razonable que obtener datos más exactos facilita las decisiones y acciones pertinentes en sitios prioritarios, tales como focalizar las remociones de individuos en sitios donde se presentó la mayor abundancia y sea correlacionado con el impacto en los arrecifes.

\section{Agradecimientos}

A la Comisión Nacional de Áreas Naturales Protegidas (CONANP), al personal de la Reserva de la Biosfera Banco Chinchorro, Parque Nacional Arrecifes de Xcalak, pescadores y a la agrupación "Jóvenes por Xcalak" que apoyaron en el monitoreo. Esta investigación fue financiada por el proyecto "Control del pez león en la RB Banco Chinchorro, PN Arrecifes de Xcalak y sus zonas de influencia"-Programa de Conservación de Especies en Riesgo (PROCER 2014). Agradecemos a Irving ChávezEstrada por su apoyo en la edición de la figura.

\section{LITERATURA CITADA}

Akins JL, J Morris \& SJ Green. 2014. In situ tagging technique for fishes provides insight into growth and movement of invasive lionfish. Ecology and Evolution 4(19): 3768-3777.

Albins MA. 2015. Invasive Pacific lionfish Pterois volitans reduce abundance and species richness of native Bahamian coral-reef fishes. Marine Ecology Progress Series 522: 231-243.

Almada-Villela PC, PF Sale, G Gold-Bouchot \& B Kjerfve. 2003. Manual de métodos para el programa de monitoreo sinóptico del SAM. Documento Técnico del SAM 4: 1-149, Belice.

Anderson LG, JK Chapman, D Escontrela \& CLA Gough. 2017. The role of conservation volunteers in the detection, monitoring and management of invasive alien lionfish. Management of Biological Invasions 8(4): 589-598.

Andradi-Brown DA, MJA Vermeij, M Slattery, M Lesser, I Bejarano, R Appeldoorn, G Goodbody-Gringley, AD Chequer, JM Pitt, C Eddy, SR Smith, E Brokovinch, HT Pinheiro, ME Jessup \& B Shepherd. 2017a. Largescale invasion of western Atlantic mesophotic reefs by lionfish potentially undermines culling-based management. Biological Invasions 19(3): 939-954.

Andradi-Brown DA, R Grey, A Hendrix, D Hitchner, CL Hunt, E Gress, K Madej, RL Parry, C RégnierMcKellar, OP Jones, M Arteaga, AP Izaguirre, AD Rogers \& DA Exton. 2017b. Depth-dependent effects of culling-do mesophotic lionfish populations undermine current management? Royal Society Open Science 4(5): 170027. <doi: 10.1098/rsos.170027> 
Arredondo-Chávez TA, JA Sánchez-Jiménez, OG ÁvilaMorales, $\mathbf{P}$ Torres-Chávez, Y Herrerias-Diego, M Medina-Nava, X Madrigal-Guridi, A Campos-Mendoza, O Domínguez-Domínguez \& JA Caballero-Vázquez. 2016. Spatio-temporal variation in the diet composition of red lionfish, Pterois volitans (Actinopterygii: Scorpaeniformes: Scorpaenidae), in the Mexican Caribbean: Insights into the ecological effect of the alien invasion. Acta Ichthyologica Et Piscatoria 46(3): 185-200.

Arias-González JE. 1998. Trophic models of protected and unprotected coral reef ecosystems in the south of the Mexican Caribbean. Journal of Fish Biology 53(suppl. A): 236-255.

Arias-González JE, C González-Gándara, JL Cabrera \& V Christensen. 2011. Predicted impact of the invasive lionfish Pterois volitans on the food web of a Caribbean coral reef. Environmental Research 111(7): 917-925.

Azzurro E, A Pais, P Consoli \& F Andaloro. 2007. Evaluating day-night changes in shallow Mediterranean rocky reef fish assemblages by visual census. Marine Biology 151(6): 2245-2253.

Bacheler NM, NR Geraldi, ML Burton, RC Muñoz \& GT Kellison. 2017. Comparing relative abundance, lengths, and habitat of temperate reef fishes using simultaneous underwater visual census, video and trap sampling. Marine Ecology Progress Series 574: 141-155.

Bogdanoff AK, J Mostowy, J Peake, CA Layman, A Brito, C González, N Hernández, DT Martínez, MR Bravo \& JA Morris. 2018. A brief description of invasive lionfish (Pterois sp.) diet composition in the Arrecifes de Cozumel National Park. Food Webs 17: e00104. <https://doi. org/10.1016/j.fooweb.2018.e00104>

Brock RE. 1982. A critique of the visual census method for assessing coral reef fish populations. Bulletin of Marine Science 32(1): 269-276.

Claydon JB, MC Calosso \& SB Traiger. 2012. Progression of invasive lionfish in seagrass, mangrove and reef habitats. Marine Ecology Progress Series 448: 119-129.

Cobián-Rojas D, JJ Schmitter-Soto, AAguilar-Perera, CM Aguilar, MA Ruiz-Zárate, G González, PP Chevalier, A García, $\mathbf{R}$ Herrera, $S$ Perera, H Caballero \& E de la Guardia. 2018. Diversidad de las comunidades de peces en dos áreas marinas protegidas del Caribe y su relación con el pez león. Revista de Biología Tropical 66(1): 189-203.

Côté IM, SJ Green \& MA Hixon. 2013. Predatory fish invaders: Insights from Indo-Pacific lionfish in the western Atlantic and Caribbean. Biological Conservation 164: 50-61.

Cure K, CE Benkwitt, TL Kindinger, EA Pickering, TJ Pusack, JL McIlwain \& MA Hixon. 2012. Comparative behavior of red lionfish Pterois volitans on native Pacific versus invaded Atlantic coral reefs. Marine Ecology Progress Series 467: 181-192.

Cure K, JL McIlwain \& MA Hixon. 2014. Habitat plasticity in native Pacific red lionfish Pterois volitans facilitates successful invasion of the Atlantic. Marine Ecology Progress Series 506: 243-253.

Darling ES, SJ Green, JK O'Leary \& IM Côté. 2011. IndoPacific lionfish are larger and more abundant on invaded reefs: a comparison of Kenyan and Bahamian lionfish Populations. Biological Invasions 13(9): 2045-2051.
Feary DA, JE Cinner, NAJ Graham \& FA JanuchowskiHartley. 2011. Effects of customary marine closures on fish behavior, spear-fishing success, and underwater visual surveys. Conservation Biology 25(2): 341-349.

Frazer TK, CA Jacoby, MA Edwards, SC Barry \& CM Manfrino. 2012. Coping with the lionfish invasion: can targeted removals yield beneficial effects? Reviews in Fisheries Science 20(4): 185-191.

García-Rivas MC, S Machkour-M'Rabet, G PérezLachaud, JJ Schmitter-Soto, C Doneys, N St-Jean, D Cobián \& Y Hénaut. 2017. What are the characteristics of lionfish and other fishes that influence their association in diurnal refuges? Marine Biology Research 13(8): 888-898.

García-Salgado M, T Camarena, G Gold, M Vásquez, G Galland, G Nava, G Alarcón \& V Ceja. 2006. Línea Base del estado del Sistema Arrecifal Mesoamericano. Documento Técnico del SAM 18: 1-168, Belize.

Gómez-Lozano R, L Anderson, JL Akins, DSA Buddo, G García-Moliner, F Gourdin, M Laurent, C Lilyestrom, JA Morris, N Ramnanan \& R Torres. 2013. Estrategia regional para el control del pez león invasor en el gran Caribe, 32 pp. Iniciativa Internacional sobre los Arrecifes Coralinos, México.

Green SJ. 2012. Monitoring: an essential action. In: Morris JA (ed). Invasive lionfish: A guide to control and management. Gulf and Caribbean Fisheries Institute Special Publication Series 1: 51-71, Marathon, Florida.

Green SJ, JLAkins \& IM Côté. 2011. Foraging behavior and prey consumption in the Indo-Pacific lionfish on Bahamian coral reefs. Marine Ecology Progress Series 433: 159-167.

Green SJ, N Tamburello, SE Miller, JL Akins \& IM Côté. 2013. Habitat complexity and fish size affect the detection of Indo-Pacific lionfish on invaded coral reefs. Coral Reefs 32(2): 413-421.

Guzmán-Méndez IA, R Rivera-Madrid, P Díaz-Jaimes, MC García-Rivas, M Aguilar-Espinosa \& JE AriasGonzález. 2017. First genetically confirmed record of the invasive devil firefish Pterois miles (Bennett, 1828) in the Mexican Caribbean. BioInvasions Records 6(2): 99-103.

Hackerott S, A Valdivia, SJ Green, IM Côté, CE Cox, JL Akins, CA Layman, WF Precht \& JF Bruno. 2013. Native predators do not influence invasion success of Pacific lionfish on Caribbean reefs. PloS ONE 8(7): e68259. $<$ https://doi.org/10.1371/journal.pone.0068259>

Hackerott S, A Valdivia, CE Cox, NJ Silbiger \& JF Bruno. 2017. Invasive lionfish had no measurable effect on prey fish community structure across the Belizean Barrier Reef. PeerJ 5:e3270.<https://doi.org/10.7717/peerj.3270>

HRI. 2012. Reporte de la salud ecológica del arrecife Mesoamericano. Una evaluación de la salud de los ecosistemas, $24 \mathrm{pp}$. Arrecifes Saludables para Gente Saludable. Iniciativa Arrecifes Saludables $<$ http://www. healthyreefs.org/cms/wp-content/uploads/2012/12/ Reporte-2012.pdf >

Kayal M, P Bosserelle \& M Adjeroud. 2017. Bias associated with the detectability of the coral-eating pest crown-ofthorns seastar and implications for reef management. Royal Society Open Science 4(8): 170396.<doi: 10.1098/ rsos.170396>

Kramer PA \& PR Kramer. 2002. Ecoregional conservation planning for the Mesoamerican Caribbean Reef, 140 pp. World Wildlife Fund, Washington. 
Kulbicki M, J Beets, P Chabanet, K Cure, E Darling, SR Floeter, R Galzin, A Green, M Harmelin-Vivien, MA Hixon, Y Letourneur, TL Loma, T McClanahan, J McIlwain, G MouTham, R Myers, JK O'Leary, S Plans, L Vigliola \& L Wantiez. 2012. Distributions of Indo-Pacific lionfishes Pterois spp. in their native ranges: implications for the Atlantic invasion. Marine Ecology Progress Series 446: 189-205.

Lang JC, KW Marks, PR Kramer, PA Kramer \& RN Ginsburg. 2012. Protocolos AGRRA version 5.5, 44 pp. Ocean Research \& Education <http://www.agrra.org/ method/AGRRA-V5.4_2010.pdf $>$

Peiffer F, S Bejarano, GP de Witte \& C Wild. 2017. Ongoing removals of invasive lionfish in Honduras and effect on native Caribbean prey fishes. PeerJ 5:e3818. <https://doi. org/10.7717/peerj.3818>

Reyes-Bonilla H, G Ramírez-Ortíz, MD Herrero-Pérezrul \& LE Calderón-Aguilera. 2016. Subestimación de la abundancia del pepino café Isostichopus fuscus (Holothuroidea: Echinodermata) en muestreos diurnos con respecto a nocturnos en el golfo de California. Revista Mexicana de Biodiversidad 87(2): 519-522.

Risk MJ. 1972. Fish diversity on a coral reef in the Virgin Islands. Atoll Research Bulletin 153: 1-6.

Rocha LA, CR Rocha, CC Baldwin, LA Weigt \& M McField. 2015. Invasive Lionfish preying on critically endangered reef fish. Coral Reefs 34(3): 803-806.

Rodríguez C, M González, C González, A Rivas, F Gourdin, R Gómez, R Torres, J Ordóñez \& V Dávila. 2014. Estrategia regional para el control del pez león en el Sistema Arrecifal Mesoamericano (SAM), 53 pp. Fondo para el Sistema Arrecifal Mesoamericano, Guatemala. <http:// www.car-spaw-rac.org/IMG/pdf/mar_subestrategia_ regional_para_el_control_del_pez_lecn.pdf $>$

Ruttenberg BI, PJ Schofield, JL Akins, A Acosta, MW Feeley, J Blondeau, SG Smith \& JS Ault. 2012. Rapid invasion of Indo-Pacific lionfishes (Pterois volitans and Pterois miles) in the Florida Keys, USA: evidence from multiple pre- and post-invasion data sets. Bulletin of Marine Science 88(4): 1051-1059.

Sabido-Itzá MM, JM Gómez-Poot, A Medina-Quej, MC García-Rivas \& W Hadad-López. 2012. Dinámica poblacional del pez león (Pterois volitans) en el Parque Nacional Arrecifes de Xcalak (PNAX), Quintana Roo, Caribe Mexicano. Proceedings of the Gulf and Caribbean Fisheries Institute 64: 67-74.
Sabido-Itzá MM, A Aguilar-Perera \& A Medina-Quej. 2016a. Length-weight and length-length relations, and relative condition factor of red lionfish, Pterois volitans (Actinopterygii: Scorpaeniformes: Scorpaenidae), from two Natural Protected Areas in the Mexican Caribbean. Acta Ichthyologica Et Piscatoria 46(4): 279-285.

Sabido-Itzá MM, A Medina-Quej, A de Jesús-Navarrete, JM Gómez-Poot, MC García-Rivas. 2016b. La estructura de tallas como evidencia del establecimiento de Pterois volitans (Scorpaeniformes: Scorpaenidae) en el sur del Caribe mexicano. Revista de Biología Tropical 64(1): 369-378.

Sabido-Itzá MM \& MC García-Rivas. 2019. Record of abundance, spatial distribution and gregarious behavior of invasive lionfish Pterois spp. (Scorpaeniformes: Scorpaenidae) in coral reefs of Banco Chinchorro Biosphere Reserve, southeastern Mexico. Latin American Journal of Aquatic Research 47(2): 349-355.

Samoilys MA \& G Carlos. 2000. Determining methods of underwater visual census for estimating the abundance of coral reef fishes. Environmental Biology of Fishes 57: 289-304.

Sanderson L \& AC Solonsky. 1986. Comparison of a rapid visual and a strip transect technique for censuring reef fish assemblages. Bulletin of Marine Science 39: 119-129.

Schofield PJ. 2010. Update on geographic spread of invasive lionfishes (Pterois volitans [Linnaeus, 1758] and P. miles [Bennett, 1828]) in the Western North Atlantic Ocean, Caribbean Sea and Gulf of Mexico. Aquatic Invasions 5(Suppl. 1): S117-S122.

Tilley A, E Carter, S Wassermann \& J López-Angarita. 2016. Enhancing management effectiveness of invasive lionfish using distance sampling and detection probability. Aquatic Conservation: Marine and Freshwater Ecosystems 26(2): 279-288.

Usseglio P, JD Selwyn, AM Downey-Wall \& JD Hogan. 2017. Effectiveness of removals of the invasive lionfish: how many dives are needed to deplete a reef? PeerJ 5:e3043. $<$ https://doi.org/10.7717/peerj.3043>

Willis TJ. 2001. Visual census methods underestimate density and diversity of cryptic reef fishes. Journal of Fish Biology 59: 1408-1411.

Zar JH. 1999. Biostatistical analysis, 663 pp. Prentice Hall, Upper Saddle River. 